\title{
Curious q-Series as Counterexamples in Padé Approximation
}

\author{
D. S. Lubinsky
}

\begin{abstract}
Basic hypergeometric, or $q$-series, are usually investigated when $|q|<1$. Less common is the case $|q|>1$, and the case where $q$ is on the unit circle is extremely rare. It is the latter curious, exotic, choice of $q$ that has yielded a number of interesting examples and counterexamples in Padé approximation, including a counterexample to the Baker-Gammel-Wills Conjecture. We survey some of these, and also pose a number of problems involving $q$-series for $q$ on the unit circle.
\end{abstract}

\section{Introduction}

Let

$$
f(z)=\sum_{j=0}^{\infty} a_{j} z^{j}
$$

be a formal power series, with complex coefficients. Given integers $m, n \geq 0$, the $(m, n)$ Padé approximant to $f$ is a rational function

$$
[m / n]=P / Q
$$

where $P, Q$ are polynomials of degree at most $m, n$ respectively, such that $Q$ is not identically 0 , and such that

$$
(f Q-P)(z)=O\left(z^{m+n+1}\right) .
$$

By this last relation, we mean that the coefficients of $1, z, z^{2}, \ldots, z^{m+n}$ in the formal power series on the left-hand side vanish. The basic idea is that $[m / n]$ is a rational function with given upper bounds on its numerator and denominator degrees, chosen in such a way that its Maclaurin series reproduces as many terms as possible in the power series $f$. It is not difficult to see that $[m / n]$ exists and is unique. 
Because there are two parameters $m$ and $n$, it is natural to form the array or Padé table

$$
\begin{array}{ccccc}
{[0 / 0]} & {[0 / 1]} & {[0 / 2]} & {[0 / 3]} & \ldots \\
{[1 / 0]} & {[1 / 1]} & {[1 / 2]} & {[1 / 3]} & \ldots \\
{[2 / 0]} & {[2 / 1]} & {[2 / 2]} & {[2 / 3]} & \ldots \\
{[3 / 0]} & {[3 / 1]} & {[3 / 2]} & {[3 / 3]} & \ldots \\
\vdots & \vdots & \vdots & \vdots & \ddots
\end{array}
$$

and then to investigate convergence of sequences of approximants as we traverse some path in the table.

The path traversed has a dramatic effect on the convergence properties of the sequence. For example, the first column $\{[m / 0]\}_{m=1}^{\infty}$ is nothing more than the sequence of partial sums of the MacLaurin series:

$$
[m / 0](z)=\sum_{j=0}^{m} a_{j} z^{j} .
$$

So the first column has the convergence properties of a Taylor series.

What about the $n$th column, where $n \geq 1$ ? Here $[m / n]$ is a rational function with at most $n$ poles, so cannot be expected to approximate as $m \rightarrow \infty$, a function with more than $n$ poles. That it does approximate functions with exactly $n$ poles is the de Montessus de Ballore theorem, the oldest and one of the most widely applied convergence results on Padé approximation. Here is the simplest form of the theorem [6, p. 282]:

Theorem 1.1 (De Montessus de Ballore's Theorem) Let $f$ be analytic at 0 and in the unit ball $U=\{z:|z|<1\}$, except for poles of total multiplicity $n$. Then

$$
\lim _{m \rightarrow \infty}[m / n](z)=f(z)
$$

uniformly in compact subsets of the unit ball omitting poles of $f$.

What happens if we try to approximate a function $f$ with $<n$ poles in $U$, using the sequence $\{[m / n]\}_{m=1}^{\infty}$ ? Because the approximants have "extra" poles, some of those extra poles do not know where to go. In this case, the sequence may converge or diverge. This is a whole topic on its own, the so-called "intermediate rows." See [29].

Even when the full sequence $\{[m / n]\}_{m=1}^{\infty}$ does not converge in this intermediate row case, is it possible that a subsequence converges? A. Beardon proved this true for the case $n=1$, but G. Baker and P. Graves-Morris observed that a subsequence often converges for any $n$. They obtained partial results and formulated a general conjecture [5]: 
Conjecture 1.2 (Baker-Graves-Morris Conjecture) Let $f$ be analytic at 0 and in the unit ball $U=\{z:|z|<1\}$, except for poles of total multiplicity $\ell<n$. Then there exists an increasing sequence $\mathcal{S}$ of positive integers such that

$$
\lim _{\substack{m \rightarrow \infty \\ m \in \mathcal{S}}}[m / n](z)=f(z),
$$

uniformly in compact subsets of the unit ball omitting poles of $f$.

The conjecture was finally resolved by Buslaev, Goncar and Suetin [8], after the efforts of many authors. They showed that there is a function analytic in the unit ball for which the conjecture is false for $n=2$. Nevertheless, they did prove that there is a constant $\sigma_{n}>0$, independent of the function, such that some subsequence converges in $\left\{z:|z|<\sigma_{n}\right\}$ away from the poles. Using scale invariance of Padé approximants, they deduced that the Baker-Graves-Morris Conjecture is true for functions meromorphic in the whole plane, with less than $n$ poles there.

In the next section, we shall discuss how the partial theta function

$$
\sum_{j=0}^{\infty} q^{j(j-1) / 2} z^{j}
$$

gives an example of a function for which the Baker-Graves-Morris Conjecture fails for every $n \geq 2$.

Traversing a diagonal seems to be the next natural case to study. In fact, surely $[n / n]$ should be the "best" Padé approximant, as it makes full use of its rational nature? The convergence nature of the diagonal sequence is complicated and not yet fully understood. There are power series $f$ with zero radius of convergence, for which $[n / n](z)$ converges as $n \rightarrow \infty$ to a function single valued and analytic in the cut-plane $\mathbb{C} \backslash[0, \infty)$. On the other hand, Hans Wallin constructed in the early 1970's [33] an entire function $f$ for which

$$
\limsup _{n \rightarrow \infty}|[n / n](z)|=\infty
$$

for all $z \in \mathbb{C} \backslash\{0\}$. The problem in Wallin's example is that each point in the plane is a limit point of poles of $\{[n / n]\}_{n=1}^{\infty}$. These poles are called spurious poles, because they do not reflect the analytic properties of the underlying function.

About the same time, John Nuttall made a seminal discovery: the spurious poles only affect a small area. More precisely, he proved [25]:

Theorem 1.3 (Nuttall's Theorem) Let $f$ be analytic at 0 and meromorphic in $\mathbb{C}$. Then $\{[n / n]\}_{n=1}^{\infty}$ converges in measure to $f$ in compact subsets of the plane. More precisely, let $r, \varepsilon>0$ and meas denote planar Lebesgue measure. Then

$$
\text { meas }\{z:|z| \leq r \text { and }|f-[n / n]|(z)>\varepsilon\} \rightarrow 0, \quad n \rightarrow \infty .
$$


Subsequently Pommerenke [27] showed that one may replace $\varepsilon$ by $\varepsilon^{n}$, planar measure by logarithmic capacity, and allow $f$ to have singularities of logarithmic capacity 0 . In particular $f$, can have essential singularities - but not branchpoints. There are far deeper analogues of the Nuttall-Pommerenke theorem for functions with branchpoints, due to H. Stahl [30], [31]. In essence, the branchpoints determine a so-called extremal set in the plane and the approximants converge in capacity inside that extremal set.

For functions meromorphic only in $U$, there is no analogue of the NuttallPommerenke theorem: $\{[n / n]\}_{n=1}^{\infty}$ need not converge in measure or capacity in any open set within $U[20]$, [28]. Nevertheless, there are still attempts to make some positive statement in this case [22].

Even these brief remarks convey to the reader the complexity of the convergence theory, due to spurious poles. Despite this inherent problem, George Baker and his collaborators found Padé approximants to be an invaluable tool in analysing singularities of series in a variety of physical problems. They also noted that in the situations where spurious poles did arise, it nevertheless affected only a subsequence of approximants. This led them to formulate a now famous conjecture [3], [4]. We shall concentrate on the following form of it:

Conjecture 1.4 (Baker-Gammel-Wills Conjecture (1961)) Let $f$ be meromorphic in the unit ball, and analytic at 0 . There is an infinite subsequence $\{[n / n]\}_{n \in \mathcal{S}}$ of the diagonal sequence $\{[n / n]\}_{n=1}^{\infty}$ that converges uniformly in all compact subsets of the unit ball omitting poles of $f$.

The conjecture was generally disbelieved from the early 1970's, at least in the above form. It was thought to be possibly true for entire functions, or functions meromorphic in the whole plane. While the latter is still unresolved, the author recently proved a counterexample to the stated form of the Baker-Gammel-Wills Conjecture. For $q$ not a root of unity, let

$$
G_{q}(z):=\sum_{j=0}^{\infty} \frac{q^{j^{2}}}{(1-q)\left(1-q^{2}\right) \cdots\left(1-q^{j}\right)} z^{j}
$$

denote the Rogers-Ramanujan function. Moreover, let

$$
H_{q}(z):=G_{q}(z) / G_{q}(q z) .
$$

For appropriate $q$ on the unit circle, the author showed [23] that $H_{q}$ provides a counterexample. This is discussed in Section 4.

Of course, the comments above provide only a small glimpse into the Padé forest. For various perspectives on the convergence theory, including the important converse results of the Russian school, see [15], [21], [32], [34].

This paper is organised as follows: in Section 2, we discuss the partial theta function. In Section 3, we discuss the work of K. Driver on Wynn's series. In Section 4, we discuss the Rogers-Ramanujan continued fraction. Finally in Section 5, we discuss a number of unresolved questions and problems that we believe are worthwhile. 


\section{The Partial Theta Function}

Basic hypergeometric, or $q$-series, is a vast topic [1], [13], [14] and I cannot pretend that I am competent to survey even parts of it. Essentially, I am a user of a tiny part of the theory. My own interest began when the Rogers-Szegö polynomials turned up in describing the behaviour of $[m / n]$ with $m \rightarrow \infty$ and $n$ fixed, when the coefficients of the underlying power series $f$ are "smooth." Let us recall some of $q$-notation: for $n \geq j \geq 0$, the Gaussian binomial coefficient is

$$
\left[\begin{array}{c}
n \\
j
\end{array}\right]=\frac{\left(1-q^{n}\right)\left(1-q^{n-1}\right)\left(1-q^{n-2}\right) \cdots\left(1-q^{n-j+1}\right)}{\left(1-q^{j}\right)\left(1-q^{j-1}\right)\left(1-q^{j-2}\right) \cdots(1-q)} .
$$

Here if $q$ is a root of unity, it must be interpreted in a limiting sense. In particular, as $q \rightarrow 1$,

$$
\left[\begin{array}{l}
n \\
j
\end{array}\right] \rightarrow\left(\begin{array}{c}
n \\
j
\end{array}\right)
$$

The Rogers-Szegö polynomial of degree $n$ is

$$
\mathcal{G}_{n}(z)=\sum_{j=0}^{n}\left[\begin{array}{l}
n \\
j
\end{array}\right] z^{j}
$$

It is closely related to polynomials appearing in the $q$-binomial theorem, which has the form

$$
\mathcal{H}_{n}(z)=\sum_{j=0}^{n}\left[\begin{array}{l}
n \\
j
\end{array}\right] q^{j(j+1) / 2} z^{j}=\prod_{j=1}^{n}\left(1+q^{j} z\right)
$$

The Rogers-Szegö polynomial $\mathcal{G}_{n}$ also turns up in the Padé denominators for the partial theta function

$$
h_{q}(z)=\sum_{j=0}^{\infty} q^{j(j-1) / 2} z^{j}
$$

These functions bear this name because they are essentially part of the theta function

$$
\sum_{j=-\infty}^{\infty} q^{j^{2}} z^{j}
$$

The partial theta function satisfies a very simple functional relation, namely,

$$
z h_{q}(q z)=h_{q}(z)-1
$$

The following identity was established by amongst others, Wynn [35].

Lemma 2.1 Let $n \geq 1$ and let $q$ not be a $j$ th root of unity for $1 \leq j \leq n$. Let $m \geq n-1$ and let $[m / n]=P_{m n} / Q_{m n}$ denote the $(m, n)$ Padé approximant for $h_{q}$, normalized by $Q_{m n}(0)=1$. Then

$$
Q_{m n}(z)=\mathcal{G}_{n}\left(-z q^{m}\right)
$$


One proof is given in [24, p. 354 ff.]. In particular when $|q|=1$, this lemma implies that if we fix $n$, and let $m$ increase to $\infty$, all the zeros of $Q_{m n}$ will lie on circles centre 0 that contain a zero of the fixed polynomial $\mathcal{G}_{n}$. Even more, if $q$ is not a root of unity, $\left\{q^{m}\right\}_{m=1}^{\infty}$ is dense on the unit circle, and so the zeros of $\left\{Q_{m n}\right\}_{m=1}^{\infty}$ will have as their limit points precisely the circles centre 0 containing zeros of $\mathcal{G}_{n}$.

This suggests a possible counterexample to the Baker-Graves-Morris Conjecture: if $|q|=1$, then $h_{q}$ is analytic inside the unit ball. If for some $|q|=1, \mathcal{G}_{n}$ has a zero, $\alpha$ say, inside the unit ball, then every $[m / n]$ with $m \geq n-1$ will have a pole on the circle $\{z:|z|=\alpha\}$, and so no subsequence of $\{[m / n]\}_{m=1}^{\infty}$ can converge to $h_{q}$ uniformly in all compact subsets of the unit ball. It turns out that for all such $q$ and for every $n \geq 2, \mathcal{G}_{n}$ has such a zero $\alpha$, and so $h_{q}$ provides a counterexample to the Baker-Graves-Morris Conjecture for every $n \geq 2$ [24]:

Theorem 2.2 Let $|q|=1$ and $q$ not be a root of unity. Then for $n \geq 2, \mathcal{G}_{n}$ has at least one zero in the unit ball. Consequently, there does not exist a subsequence of $\{[m / n]\}_{m=1}^{\infty}$ that converges to $h_{q}$ uniformly in all compact subsets of the unit ball.

We remind the reader, as mentioned in the previous section, that this was not the first counterexample. The first was given by Buslaev, Goncar, and Suetin [8]. They showed that the function

$$
f(z)=\frac{1+2^{1 / 3} z}{1-z^{3}}
$$

has no subsequence of $\{[m / 2]\}_{m=1}^{\infty}$ converging uniformly in $\left\{z:|z| \leq 2^{-1 / 3}\right\}$. We also recall that they did show that a subsequence converges in some ball centre 0 , with the radius being independent of the underlying function.

Where is the smallest zero of $\mathcal{G}_{n}$ ? Using numerical computation, we showed [24] that for $n=2$, there exists a $q$ such that $\mathcal{G}_{2}$ has a zero with absolute value $0.58 \ldots$ and that this is smallest possible as $q$ ranges over the unit circle. As $n$ increases, the size of the smallest zero of $\mathcal{G}_{n}$ as $q$ ranges over the unit circle decreases, and reaches $0.24 \ldots$ for $n=17$.

This suggests an interesting problem, when taken in conjunction with BuslaevGoncar-Suetin's positive result:

Problem 2.3 Let $f$ be analytic in $U$ and $n \geq 2$. Let $\sigma_{n}(f)$ denote the radius of the largest disc centre 0 for which some subsequence of $\{[\mathrm{m} / n]\}_{m=1}^{\infty}$ converges uniformly in each compact subset of that disc. Compute

$$
\sigma_{n}^{*}:=\inf \left\{\sigma_{n}(f): f \text { analytic in } U\right\} .
$$

The only known lower bound for $\sigma_{n}^{*}$ is due to Buslaev, Goncar and Suetin. From the examples mentioned above, we know

$$
\sigma_{2}^{*} \leq 0.58 \ldots
$$


and

$$
\sigma_{17}^{*} \leq 0.24 \ldots
$$

There has been virtually no work on this problem, maybe because it is so difficult. But surely, it can be resolved for $n=2$ for example?

In actual fact, Ed Saff and the author were fishing for something far bigger with the partial-theta function: we had initially hoped that it would provide a counterexample to the Baker-Gammel-Wills Conjecture. As it turned out, for every $q$, the Baker-Gammel-Wills Conjecture is true for $h_{q}$. In retrospect, I understand why it cannot provide a counterexample, and this is best explained using the concept of a continued fraction.

Given a formal power series (1), we may also formally write

$$
f(z)=c_{0}+\frac{c_{1} z^{k_{1}}}{1+\frac{c_{2} z^{k_{2}}}{1+\frac{c_{3} z^{k_{3}}}{{ }_{1+}}}},
$$

or more compactly,

$$
f(z)=c_{0}+\frac{c_{1} z^{k_{1}} \mid}{\mid 1}+\frac{c_{2} z^{k_{2}} \mid}{\mid 1}+\frac{c_{3} z^{k_{3}} \mid}{\mid 1}+\cdots
$$

where $\left\{c_{j}\right\}$ are complex numbers, and $\left\{k_{j}\right\}$ are positive integers. This is called the $C$-fraction corresponding to $f$. In the case that all $k_{j}=1$ (a most desirable phenomenon), the $C$-fraction is said to be normal.

Just as we define the value of an infinite series to be the limit of the sequence of partial sums (each of which is a truncation of the series), so we define the value of a continued fraction to be the limit of the sequence formed by successive truncations of it. For $n \geq 1$, let

$$
\frac{\mu_{n}}{\nu_{n}}(z)=c_{0}+\frac{c_{1} z^{k_{1}} \mid}{\mid 1}+\frac{c_{2} z^{k_{2}} \mid}{\mid 1}+\frac{c_{3} z^{k_{3}} \mid}{\mid 1}+\cdots+\frac{c_{n} z^{k_{n}} \mid}{\mid 1} .
$$

This is called the $n$th convergent of the continued fraction; it is a rational function of $z$. We define the value of the continued fraction to be

$$
\lim _{n \rightarrow \infty} \frac{\mu_{n}}{\nu_{n}}(z),
$$

if this limit exists.

There is a close relationship between continued fractions and Padé approximants [19]. In particular, in the normal case, where all $k_{j}=1$, the sequence of convergents $\left\{\mu_{n} / \nu_{n}\right\}_{n=1}^{\infty}$ comprises the main diagonal $\{[n / n]\}_{n=1}^{\infty}$ and the superdiagonal $\{[n+1 / n]\}_{n=1}^{\infty}$. Thus we can use continued fraction techniques to study Padé approximants, and conversely, Padé methods give some insight into continued fractions. 
For the partial theta function $h_{q}$, the continued fraction has the form

$$
h_{q}(z)=1+\frac{z \mid}{\mid 1}+\frac{-q z \mid}{\mid 1}+\frac{q(1-q) z \mid}{\mid 1}+\frac{-q^{3} z \mid}{\mid 1}+\frac{q^{2}\left(1-q^{2}\right) z \mid}{\mid 1}+\cdots
$$

So the continued fraction is normal. For $|q|=1$, we see that the coefficients of $z$ are bounded in absolute value by 2 . An old theorem of Worpitzky [19] then ensures that the continued fraction converges at least for $|z| \leq \frac{1}{8}$. However, the coefficients are also oscillatory, some taking the form $-q^{n}$ and others taking the form $q^{n}\left(1-q^{n}\right)$, $n \geq 1$. This prevents application of standard convergence theorems for continued fractions beyond the range of $z$ covered by Worpitzky's theorem. It also suggests that convergence of the full sequence of convergents, throughout the unit ball $U$, may not take place.

Indeed, for a given $q$, let $\Delta_{q}$ denote the inf of the absolute values of the zeros of $\mathcal{G}_{n}$, so that

$$
\Delta_{q}=\inf \left\{|z|: \mathcal{G}_{n}(z)=0, \text { some } n \geq 1\right\} .
$$

We know that $\Delta_{q}<1$. It was shown in [24] that the continued fraction converges in $\left\{z:|z|<\Delta_{q}\right\}$ but not in any larger disk. Nevertheless, as Ed Saff and I found to our disappointment, some subsequence of the convergents does converge throughout the unit ball to $h_{q}$, and then (with a little more work) also some subsequence of $\{[n / n]\}_{n=1}^{\infty}$. That subsequence corresponded to an infinite sequence $\mathcal{S}$ of integers for which

$$
q^{n} \rightarrow 1, \quad n \rightarrow \infty, \quad n \in \mathcal{S}
$$

For this subsequence the coefficients

$$
q^{n}\left(1-q^{n}\right) \rightarrow 0
$$

which was sufficient to guarantee convergence. In retrospect, it should not be surprising that such a subsequence yields good convergents: recall the theorem that when the full sequence of continued fraction coefficients converges to 0 , the continued fraction converges to a meromorphic function in the whole complex plane (except at the poles).

There were many other fascinating features of the partial theta function, and of its Padé approximants. But undoubtedly the most significant is that for each $|q|=1$ with $q$ not a root of unity, $h_{q}$ provides a counterexample to the BakerGraves-Morris Conjecture for every $n \geq 2$.

\section{Wynn's Series}

While the Padé approximants for the partial theta function failed to provide a counterexample to the Baker-Gammel-Wills Conjecture, their curious and irregular behaviour suggested that a counterexample might well be found in some close 
cousin. Certainly it seems a good idea to use formulas for $q$-series not in the usual setting, namely $|q|<1$, but in the exotic domain $|q|=1$, with the hope that something truly pathological might arise.

Of course one needs explicit formulas if any analysis is to be possible, and it was the 1967 work of P. Wynn [35] that suggested the next candidates for study. To some extent, Wynn's work overlapped with earlier work of Heine, Balk, Gragg. In that paper, Wynn considered three classes of series.

(I)

$$
\begin{aligned}
f_{1}(z) & =\sum_{j=0}^{\infty}\left[\prod_{k=0}^{j-1}\left(A-q^{k+\alpha}\right)\right] z^{j} \\
& ={ }_{2} \Phi_{1}\left(\begin{array}{c}
A^{-1} q^{\alpha}, q ; q, A z \\
0
\end{array}\right),
\end{aligned}
$$

in the language of basic hypergeometric series. Here $A, q \in \mathbb{C}, \alpha \in \mathbb{R}$ and we assume that

$$
A \neq q^{k+\alpha}, \quad k \geq 0,
$$

so that the series does not terminate. The functional relation is [10]

$$
f_{1}(z)(1-z A)=1-f_{1}(q z) z q^{\alpha} .
$$

Note that if $A=\alpha=0, f_{1}$ reduces essentially to the partial theta function.

(II)

$$
\begin{aligned}
f_{2}(z) & =\sum_{j=0}^{\infty}\left[\prod_{k=0}^{j-1}\left(\frac{1}{C-q^{k+\gamma}}\right)\right] z^{j} \\
& ={ }_{2} \Phi_{1}\left(\begin{array}{c}
q, 0 ; q, C^{-1} z \\
C^{-1} q^{\gamma}
\end{array}\right),
\end{aligned}
$$

where $C, q \in \mathbb{C}, \gamma \in \mathbb{R}$ and we assume that

$$
C \neq q^{k+\gamma}, \quad k \geq 0
$$

The functional relation is [11]

$$
f_{2}(z)(C-z)=C-q^{\gamma-1}+q^{\gamma-1} f_{2}(q z) .
$$

$$
\begin{aligned}
f_{3}(z) & =\sum_{j=0}^{\infty}\left[\prod_{k=0}^{j-1}\left(\frac{A-q^{k+\alpha}}{C-q^{k+\gamma}}\right)\right] z^{j} \\
& ={ }_{2} \Phi_{1}\left(\begin{array}{c}
A^{-1} q^{\alpha}, q ; q, A C^{-1} z \\
C^{-1} q^{\gamma}
\end{array}\right),
\end{aligned}
$$


where $A, C, q \in \mathbb{C} ; \alpha, \gamma \in \mathbb{R}$ and we assume that (3) and (4) hold. The functional relation is [12]

$$
f_{3}(z)(C-z A)=C-q^{\gamma-1}+f_{3}(q z)\left(q^{\gamma-1}-z q^{\alpha}\right) .
$$

In all three cases, the functional relation is a useful tool in investigating the analytic properties of the function. Let us look at $f_{3}$. In her thesis, K. A. Driver [9] proved, amongst other things, the following: let $A, C \neq 0,|A|,|C| \neq 1$, let $|q|=1$ and $q$ not be a root of unity. Then $f_{3}$ has radius of convergence

$$
R=\left[\max \{1,|A|\} \min \left\{1,|C|^{-1}\right\}\right]^{-1},
$$

and if

$$
C \neq A q^{\gamma-\alpha+j}, \quad j=0,1,2, \ldots,
$$

$f_{3}$ has a natural boundary on its circle of convergence. (If this last condition fails, $f_{3}$ is a rational function.) The continued fraction has the form

$$
f_{3}(z)=1+\frac{c_{1} z \mid}{\mid 1}+\frac{c_{2} z \mid}{\mid 1}+\frac{c_{3} z \mid}{\mid 1}+\cdots,
$$

where

$$
c_{2 n+1}=\frac{q^{2 n-1}\left(1-q^{n}\right)\left(A q^{\gamma}-C q^{1-n+\alpha}\right)}{\left(C-q^{2 n+\gamma}\right)\left(C-q^{2 n+\gamma-1}\right)}
$$

and

$$
c_{2 n}=\frac{-q^{n-1}\left(A-q^{n+\alpha}\right)\left(C-q^{n+\gamma-1}\right)}{\left(C-q^{2 n+\gamma-1}\right)\left(C-q^{2 n+\gamma-2}\right)} .
$$

A detailed analysis was provided of the continued fraction. In particular, Driver proved that the full sequence of convergents (and hence $\{[n / n]\}_{n=1}^{\infty}$ ) converges in measure and in capacity in compact subsets of $\{z:|z|<R\}$. Moreover, some subsequence does converge uniformly in compact subsets of that ball. In fact such a subsequence corresponds to the infinite sequence of integers $\mathcal{S}$ satisfying (2), just as for the partial theta function.

Thus again hopes of a counterexample to the Baker-Gammel-Wills Conjecture dissipated, although there were a host of other interesting features.

\section{The Rogers-Ramanujan Continued Fraction}

For $q$ not a root of unity, let

$$
G_{q}(z):=\sum_{j=0}^{\infty} \frac{q^{j^{2}}}{(1-q)\left(1-q^{2}\right) \cdots\left(1-q^{j}\right)} z^{j}
$$


denote the Rogers-Ramanujan function. (At this stage, it is a formal power series.) It admits the functional relation

$$
G_{q}(z)=G_{q}(q z)+q z G_{q}\left(q^{2} z\right) .
$$

Moreover, let

$$
H_{q}(z):=G_{q}(z) / G_{q}(q z) .
$$

From the functional relation for $G_{q}$, it is easy to derive one for $H_{q}$ :

$$
H_{q}(z)=1+\frac{q z}{H_{q}(q z)} .
$$

Iterating this leads to

$$
H_{q}(z)=1+\frac{q z}{1+\frac{q^{2} z}{1+\cdot \frac{q^{n} z}{H_{q}\left(q^{n} z\right)}}}
$$

and hence to the formal infinite continued fraction

$$
H_{q}(z)=1+\frac{q z \mid}{\mid 1}+\frac{q^{2} z \mid}{\mid 1}+\frac{q^{3} z \mid}{\mid 1}+\cdots .
$$

For $|q|<1$, the continued fraction was considered independently by L. J. Rogers and S. Ramanujan in the early part of the twentieth century.

There are several differences between the Rogers-Ramanujan continued fraction (c.f.), and those from Wynn's series. Firstly, if $|q|=1$, all the coefficients in the Rogers-Ramanujan c.f. have modulus 1 , whereas a subsequence of the coefficients in the c.f. for Wynn's series converges to 0 . Moreover the latter subsequence is associated with a subsequence of the convergents to the c.f. that converges throughout the region of analyticity. This already suggests that there may not be a uniformly convergent subsequence of the convergents for the Rogers-Ramanujan c.f. Secondly, in the case where $q$ is a root of unity, all of the Wynn's series reduce to rational functions, while the Rogers-Ramanujan c.f. corresponds to a function with branchpoints.

We see that the radius of convergence of $G_{q}$ is

$$
R(q):=\liminf _{j \rightarrow \infty}\left|\prod_{k=0}^{j-1}\left(1-q^{k}\right)\right|^{1 / j} .
$$

It was essentially proved in [16] that

$$
R(q)=\liminf _{j \rightarrow \infty}\left|1-q^{j}\right|^{1 / j} .
$$


If we write $q=e^{2 \pi i \tau}$, this is readily reformulated in terms of the diophantine approximation properties of $\tau$. Since $\left|1-q^{j}\right|=2|\sin [\pi(j \tau-k)]|$ for any integer $k$, we see that

$$
R(q)=\liminf _{j \rightarrow \infty}\|j \tau\|^{1 / j},
$$

where $\|x\|$ denotes the distance from $x$ to the nearest integer. In particular, elementary diophantine approximation theory shows that for a.e. $q$ on the unit circle, $R(q)=1$.

Using the functional relation, one can show that $G_{q}$ has a natural boundary on its circle of convergence. Then $H_{q}$ is meromorphic inside this ball. One can also show that $H_{q}$ has a natural boundary on its circle of meromorphy, that is on the largest circle centre 0 , inside which it is meromorphic. This does not follow from the fact that $G_{q}$ has a natural boundary on $\{z:|z|=R(q)\}$, and must be proved independently from the functional relation for $H_{q}$.

Far more curious, is the fact that the natural boundary of $H_{q}$ need not coincide with that of $G_{q}$ : somehow in the division in (5), the natural boundary of $G_{q}$ "cancels out" [23]:

Theorem 4.1 Let $0<\sigma<\frac{1}{4}$. Then there exists $|q|=1$, with $q$ not a root of unity, such that $G_{q}$ is analytic in $\{z:|z|<\sigma\}$ and has a natural boundary on $\{z:|z|=\sigma\}$. However, if we define $H_{q}$ by (5), then it may be continued meromorphically to $\{z:|z|<\rho\}$, where $\rho \geq \frac{1}{4}>\sigma$. Thus $H_{q}$ is meromorphic in $\{z:|z|<\rho\}$, and has a natural boundary on $\{z:|z|=\rho\}$.

This is the first time that this author has seen a natural boundary cancel out: we are all familiar with poles that cancel, but natural boundaries?

Problem 4.2 Explain this cancellation.

This phenomenon is unusual. Indeed, for a.e. $q$ on the unit circle, $G_{q}$ is analytic in the unit ball $U$ with natural boundary on the unit circle, and $H_{q}$ is meromorphic in the unit ball, with natural boundary on the unit circle.

Proofs of the explicit formulae for the numerator and denominator polynomials $\mu_{n}$ and $\nu_{n}$ in the convergent

$$
\frac{\mu_{n}(z)}{\nu_{n}(z)}=1+\frac{q z \mid}{\mid 1}+\frac{q^{2} z \mid}{\mid 1}+\cdots+\frac{q^{3} z \mid}{\mid 1}
$$

were first published by M. Hirschorn in 1972 [17]:

$$
\mu_{n}(z)=\sum_{k=0}^{\left[\frac{n+1}{2}\right]} z^{k} q^{k^{2}}\left[\begin{array}{c}
n+1-k \\
k
\end{array}\right]
$$

and

$$
\nu_{n}(z)=\mu_{n-1}(q z)=\sum_{k=0}^{\left[\frac{n}{2}\right]} z^{k} q^{k(k+1)}\left[\begin{array}{c}
n-k \\
k
\end{array}\right],
$$


where $[x]$ is the greatest integer $\leq x$. The author met Hirschhorn in Sydney in 2000, and was intrigued by the story of these identities. Like so many other $q$-identities, they appeared in notes of Ramanujan, but without proof. Moreover, Hirschhorn was not aware of Ramanujan's notes at the time he wrote his papers - again a common occurrence.

In describing the behaviour of $\left\{\mu_{n}\right\}$ and $\left\{\nu_{n}\right\}$, we need an elementary observation from number theory: if $q$ is not a root of unity, then $\left\{q^{n}\right\}_{n=1}^{\infty}$ is dense on the unit circle, and one may extract a subsequence converging to an arbitrary $\beta$ on the unit circle. This helps to introduce our main convergence theorem for the convergents (recall that $R(q)$ is the radius of convergence of $G_{q}$ ) :

Theorem 4.3 Let $|q|=1$, and $q$ not be a root of unity. Let $|\beta|=1$ and $\mathcal{S}$ be any infinite sequence of positive integers with

$$
\lim _{n \rightarrow \infty, n \in \mathcal{S}} q^{n}=\beta .
$$

Then uniformly in compact subsets of $\{z:|z|<R(q)\}$,

$$
\begin{aligned}
& \lim _{n \rightarrow \infty, n \in \mathcal{S}} \mu_{n}(z)=\overline{G_{q}(\overline{\beta q z})} G_{q}(z) ; \\
& \lim _{n \rightarrow \infty, n \in \mathcal{S}} \nu_{n}(z)=\overline{G_{q}(\overline{\beta q z})} G_{q}(q z) ;
\end{aligned}
$$

and uniformly in compact subsets of $\{z:|z|<R(q)\}$ omitting zeros of $G_{q}(\overline{\beta q z})$ and $G_{q}(q z)$,

$$
\lim _{n \rightarrow \infty, n \in \mathcal{S}} \frac{H_{q}(z)-\frac{\mu_{n}(z)}{\nu_{n}(z)}}{(-1)^{n} z^{n+1} q^{(n+1)(n+2) / 2}}=\frac{G_{q}\left(\beta q^{2} z\right)}{G_{q}(q z)^{2} \overline{G_{q}(\overline{\beta q z})}}
$$

and so in such sets omitting these zeros,

$$
\lim _{n \rightarrow \infty, n \in \mathcal{S}} \frac{\mu_{n}(z)}{\nu_{n}(z)}=H_{q}(z) .
$$

The crucial point in the last line is that the convergence takes place away from the zeros of both $G_{q}(z)$ and $G_{q}(\overline{\beta q z})$. The zeros of $G_{q}(\overline{\beta q z})$ need not be poles of $H_{q}$, and yet (9) shows that they attract poles of the convergents. Moreover, because $|\beta|=1$, both $G_{q}(z)$ and $\overline{G_{q}(\overline{\beta q z})}$ have the same number of zeros on any circle centre 0 , and this is true of every such $\beta$. Hence:

Corollary 4.4 Let $|q|=1$, not a root of unity. Assume that $r<R(q)$ and $H_{q}$ has poles of total multiplicity $\ell$ on $\{z:|z|=r\}$. Let $\mathcal{O}$ be an open set containing this circle. Then there exists $n_{0}$ such that for $n \geq n_{0}, \mu_{n} / \nu_{n}$ has poles of total multiplicity $\geq 2 \ell$ in $\mathcal{O}$. 
This is the first such example in the literature, in which all approximants of large order have more poles than the approximated function in a region of meromorphy. If we could show that there does not exist $\beta$ for which the zero sets of $G_{q}(q z)$ and $G_{q}(\overline{\beta q z})$ are the same, then it establishes a counterexample to the Baker-Gammel-Wills conjecture. For then, given any subsequence of the convergents, we can extract a further subsequence for which (7) holds for some $\beta$; that subsequence cannot converge uniformly in a compact set containing zeros of $G_{q}(\overline{\beta q z})$ that are not zeros of $G_{q}(z)$.

A little thought shows that the zero sets of $G_{q}(q z)$ and $G_{q}(\overline{\beta q z})$ are not the same for any $|\beta|=1$, iff the zeros of $G_{q}$ are not symmetric about any line through 0 . Thus:

Corollary 4.5 Let $|q|=1$, and $q$ not be a root of unity. Assume that the zeros of $G_{q}$ inside its circle of convergence are not symmetric about any line through 0 . Then $H_{q}$ provides a counterexample to the Baker-Gammel-Wills Conjecture.

Intuitively, there was a lot of reason to believe in the desired asymmetry, at least from the following standpoint: recall that a Maclaurin series with real coefficients has zeros symmetric about the real axis, that is, they occur in conjugate pairs. Conversely, one might hope that for special functions, zeros that occur in conjugate pairs are associated with Maclaurin series with real coefficients. After a rotation of the variable, symmetry of zeros of $G_{q}$ about some line through 0 , would become symmetry about the real axis. Yet the arguments of the coefficients of $G_{q}(\gamma z)$ are highly oscillatory for any $\gamma$ on the unit circle, and there is no reason to expect symmetry.

For a long time, the author tried to prove this asymmetry property, but failed. Since numerical computation might provide some insight, the author was fortunate to be able to ask A. Knopfmacher (who is, amongst other things, a Mathematica expert) to plot some zeros a few years ago. Of course, we cannot easily compute $G_{q}$ itself, but we can with reasonable accuracy, plot the zeros of the partial sums

$$
S_{m, q}(z)=\sum_{j=0}^{m} \frac{q^{j^{2}}}{(1-q)\left(1-q^{2}\right) \cdots\left(1-q^{j}\right)} z^{j} .
$$

In the case when the radius of convergence is $R(q)=1$, the partial sums converge rapidly within the unit ball to $G_{q}$ as $m \rightarrow \infty$, and so their zeros should approximate the zeros of $G_{q}$ well inside the unit circle.

We typically chose [18]

$$
q=\exp (2 \pi i / \sqrt{\ell})
$$

where $\ell$ is some positive integer. For almost all the choices of $\ell, S_{m, q}$ had zeros asymmetric with respect to any line through 0 . Moreover, as we increased $m$ from 10 through to 100 , the zeros well within the unit circle remained the same. In 
fact, in almost all cases, the two zeros closest to the origin had distinct modulus and distinct argument, which is already enough to establish asymmetry. This very strongly suggested that we do have a counterexample. However, it took some time to find a choice of $q$ for which I could obtain a sufficiently fine estimate of

$$
G_{q}-S_{m, q}
$$

to turn this into a proof. This was finally done for a special $q$ in January 2001 [23]:

Theorem 4.6 Let

$$
q:=\exp (2 \pi i \tau)
$$

where

$$
\tau:=\frac{2}{99+\sqrt{5}} .
$$

Then $H_{q}$ is meromorphic in the unit ball and analytic at 0 . There does not exist any subsequence of $\left\{\mu_{n} / \nu_{n}\right\}_{n=1}^{\infty}$ that converges uniformly in all compact subsets of

$$
\mathcal{A}:=\{z:|z|<0.46\}
$$

omitting poles of $H_{q}$. In particular no subsequence of $\{[n / n]\}_{n=1}^{\infty}$ or $\{[n+1 / n]\}_{n=1}^{\infty}$ can converge uniformly in all compact subsets of $\mathcal{A}$ omitting poles of $H_{q}$.

After this counterexample was announced, it was discussed in the seminar of A. Gonchar at the Steklov Institute of Mathematics in Moscow. This inspired V. Buslaev to construct a simpler counterexample to the Baker-Gammel-Wills Conjecture, namely the algebraic function:

$$
f(z)=\frac{-27+6 z^{2}+3(9+\zeta) z^{3}+\sqrt{81\left(3-(3+\xi) z^{3}\right)^{2}+4 z^{6}}}{2 z\left(9+9 z+(9+\xi) z^{2}\right)}
$$

where

$$
\xi=-\frac{1}{2}+\frac{\sqrt{3}}{2} i
$$

He shows that this function is analytic in the unit ball, but no subsequence of $\{[n / n]\}_{n=1}^{\infty}$ can converge at one of three special points inside the unit ball. See the announcement [7].

\section{$5 \quad$ Suggested Problems}

In a complicated subject like Padé convergence theory, the resolution of the BakerGammel-Wills Conjecture raises many problems about weaker forms of the conjecture. Some of those are discussed in [23], [32]. Here we shall discuss a few problems specifically relating to $q$-series, that we believe are interesting. 
We have already mentioned the strange cancellation of the natural boundary of $G_{q}$ (Problem 4.2). But there are many others. We know, thanks to work of G. Petruska [26], that given $R \in[0,1]$, we may find $q$ such that $G_{q}$ has radius of convergence $R$, that is $R=R(q)$. But what values can the radius of meromorphy of $H_{q}$ assume? Thanks to a theorem of Worpitzky, which ensures that the continued fraction (6) converges for $|z| \leq \frac{1}{4}$, we know this radius is $\geq \frac{1}{4}$. Moreover, for almost every $q$, it is 1 , but are there any exceptional values?

Problem 5.1 Let $|q|=1$, and $q$ not be a root of unity. Let $\rho(q)$ denote the largest circle centre 0 inside which $H_{q}$ may be meromorphically continued. Can $\rho(q)$ assume any value other than 1 ? If so, what is its range of values?

Another interesting problem, is to investigate the zeros of $G_{q}$ without the use of any numerical package:

Problem 5.2 (i) Investigate the structure of zeros of $G_{q}$ when $|q|=1$ and $R(q)>0$.

(ii) Moreover, investigate whether for every such $q$, the zeros of $G_{q}$ are not symmetric about any line through 0.

(iii) Investigate the behaviour of the zero of $G_{q}$ closest to the origin as $q$ traverses the unit circle.

It is instructive here to recall that the $q$-exponential functions are

$$
\begin{aligned}
& e_{q}(z)=\sum_{j=0}^{\infty} \frac{z^{j}}{(1-q)\left(1-q^{2}\right)\left(1-q^{3}\right) \cdots\left(1-q^{j}\right)} ; \\
& E_{q}(z)=\sum_{j=0}^{\infty} \frac{q^{j(j-1) / 2} z^{j}}{(1-q)\left(1-q^{2}\right)\left(1-q^{3}\right) \cdots\left(1-q^{j}\right)} .
\end{aligned}
$$

Now in $\{z:|z|<R(q)\}$, a direct calculation shows that

$$
e_{q}(z) E_{q}(-z)=1
$$

and hence $e_{q}$ and $E_{q}$ have no zeros in that ball. In contrast, we know that

$$
G_{q}(z)=\sum_{j=0}^{\infty} \frac{q^{j^{2}} z^{j}}{(1-q)\left(1-q^{2}\right)\left(1-q^{3}\right) \cdots\left(1-q^{j}\right)}
$$

may have zeros. This suggests:

Problem 5.3 Let $|q|=1$ and $R(q)>0$. For $a \geq 0$, let

$$
G_{a, q}(z)=\sum_{j=0}^{\infty} \frac{q^{a j^{2}} z^{j}}{(1-q)\left(1-q^{2}\right)\left(1-q^{3}\right) \cdots\left(1-q^{j}\right)} .
$$

For which a $>0$ does $G_{a, q}$ have zeros? 
Of course these are very specific problems. But there are also some quite general ones, involving the mere definition of $q$-special functions. We have seen above that there is no problem with defining $q$-exponential functions for $q$ not a root of unity. But a $q$-gamma function is far more challenging. The $q$-gamma function $\Gamma_{q}(x)$ is the unique solution of the difference equation

$$
\Gamma_{q}(x+1)=\frac{q^{x}-1}{q-1} \Gamma_{q}(x),
$$

with normalisation

$$
\Gamma_{q}(1)=1,
$$

and with $\log \Gamma_{q}(x)$ convex for $x>0$. As $q \rightarrow 1$, we see that this becomes the classical relation

$$
\Gamma(x+1)=x \Gamma(x) .
$$

For $q \in(0,1)$, we have [2]

$$
\Gamma_{q}(x)=(1-q)^{1-x} \prod_{n=0}^{\infty} \frac{1-q^{n+1}}{1-q^{n+x}} .
$$

It is easily seen that this also defines a function analytic in the upper-half plane $(\operatorname{Re} x>0)$.

Problem 5.4 Define a q-gamma function for $|q|=1$.

The problem seems to be that any solution of the functional relation (10) with $|q|=1, q$ not a root of unity, cannot be defined on the real axis. Of course there are other $q$-special functions for which similar problems might arise. The definition of $q$-special functions for $q$ on the unit circle might seem like the sort of subject one invents for the sake of "creating a gap in the literature." However, the author believes that $q$-series with $|q|=1$, and $q$ not a root of unity, will have a growing number of applications. Moreover, not all of these will be in the search for pathological examples.

\section{Acknowledgement}

It was a privilege to take part in IDOMAT2001. The author thanks the organisers for all their hard work, the kind invitation, and the stimulating and well run conference.

\section{References}

[1] G. Andrews, R. Askey, R. Roy, Special Functions, Cambridge University Press, Cambridge, 1999. 
[2] R. Askey, The q-Gamma and q-Beta Functions, Applicable Analysis, 8(1978), $125-141$.

[3] G. A. Baker, Essentials of Padé Approximants, Academic Press, New York, 1975.

[4] G. A. Baker, J. L. Gammel, J. G. Wills, An Investigation of the Applicability of the Padé Approximant Method, J. Math. Anal. Appl., 2(1961), 405-418.

[5] G. A. Baker and P. R. Graves-Morris, Convergence of Rows of the Padé Table, J. Math. Anal. Appl., 57(1977), 323-339.

[6] G. A. Baker and P. R. Graves-Morris, Padé Approximants, 2nd edn., Encyclopaedia of Mathematics and its Applications, Vol. 59, Cambridge University Press, Cambridge, 1996.

[7] V. I. Buslaev, Simple Counterexample to the Baker-Gammel-Wills Conjecture, East Journal on Approximations, 4(2001), 515-517.

[8] V. I. Buslaev, A. A. Goncar, and S. P. Suetin, On Convergence of Subsequences of the mth Row of a Padé Table, Math. USSR. Sbornik, 48(1984), 535-540.

[9] K. A. Driver, Convergence of Padé Approximants for some $q$-Hypergeometric Series (Wynn's Power Series I, II, III), Thesis, University of the Witwatersrand, 1991.

[10] K. A. Driver and D. S. Lubinsky, Convergence of Padé Approximants for a qHypergeometric Series (Wynn's Power Series I), Aequationes Mathematicae, 42(1991), 85-106.

[11] K. A. Driver and D. S. Lubinsky, Convergence of Padé Approximants for a q-Hypergeometric Series (Wynn's Power Series II), Colloquia Mathematica Societatis Janos Bolyai, Vol. 58, pp. 221-239, Janos Bolyai Math Society, 1990.

[12] K. A. Driver and D. S. Lubinsky, Convergence of Padé Approximants for a qHypergeometric Series (Wynn's Power Series III), Aequationes Mathematicae, 45(1993), 1-23.

[13] N. J. Fine, Basic Hypergeometric Series and Their Applications, American Mathematical Society Mathematical Surveys and Monographs No. 27, American Mathematical Society, Providence, Rhode Island, 1988.

[14] G. Gasper and M. Rahman, Basic Hypergeometric Series, Cambridge University Press, Cambridge, 1990.

[15] A. A. Goncar, On Uniform Convergence of Diagonal Padé Approximants, Math. USSR. Sbornik, 46(1982), 539-559. 
$q$-Series as Counterexamples

[16] G. H. Hardy and J. E. Littlewood, Notes on the Theory of Series (XXIV): A Curious Power Series, Math. Proc. Cambridge Philosophical Soc., 42(1946), $85-90$.

[17] M. D. Hirschhorn, Partitions and Ramanujan's Continued Fraction, Duke Math. Journal, 39(1972), 789-791.

[18] A. Knopfmacher and D. S. Lubinsky, Mathematica Evidence that Ramanujan kills Baker-Gammel-Wills, Applied Mathematics and Computation, 128(2002), 289-302.

[19] L. Lorentzen and H. Waadeland, Continued Fractions with Applications, North Holland, 1992.

[20] D. S. Lubinsky, Diagonal Padé Approximants and Capacity, J. Math. Anal. Applns., 78(1980), 58-67.

[21] D. S. Lubinsky, Will Ramanujan kill Baker-Gammel-Wills? (A Selective Survey of Padé Approximation), (in) Proceedings of IDOMAT98, International Series in Numerical Mathematics, Vol. 132, Birkhauser, Basel, 1999, pp. 159174.

[22] D. S. Lubinsky, Weighted Maximum over Minimum Modulus of Polynomials, with Application to Ray Sequences of Padé Approximants, Constructive Approximation, 18(2002), 285-308.

[23] D. S. Lubinsky, Rogers-Ramanujan and the Baker-Gammel-Wills (Padé) Conjecture, to appear in Annals of Mathematics.

[24] D. S. Lubinsky and E. B. Saff, Convergence of Padé Approximants of Partial Theta Functions and the Rogers-Szegö Polynomials, Constr. Approx., 3(1987), 331-361.

[25] J. Nuttall, Convergence of Padé Approximants of Meromorphic Functions, J. Math. Anal. Appl., 31(1970), 147-153.

[26] G. Petruska, On the Radius of Convergence of q-Series, Indagationes Mathematicae, 3(3) (1992), 353-364.

[27] C. Pommerenke, Padé Approximants and Convergence in Capacity, J. Math. Anal. Appl., 41(1973), 775-780.

[28] E. A. Rakhmanov, On the Convergence of Padé Approximants in Classes of Holomorphic Functions, Math. Ussr. Sbornik, 40(1981), 149-155.

[29] A. Sidi, Quantitative and Constructive Aspects of the Generalized Koenig's and de Montessus's Theorems for Padé Approximants, J. Comp. Appl. Math., 29(1990), 257-291. 
[30] H. Stahl, General Convergence Results for Padé Approximants, (in) Approximation Theory VI, (eds. C. K. Chui, L. L. Schumaker, J. D. Ward), Academic Press, San Diego, 1989, pp. 605-634.

[31] H. Stahl, The Convergence of Padé Approximants to Functions with Branch Points, J. Approx. Theory, 91(1997), 139-204.

[32] H. Stahl, Conjectures Around the Baker-Gammel-Wills Conjecture: Research Problems 97-2, Constr. Approx., 13(1997), 287-292.

[33] H. Wallin, The Convergence of Padé Approximants and the Size of the Power Series Coefficients, Applicable Anal., 4(1974), 235-251.

[34] H. Wallin, Potential Theory and Approximation of Analytic Functions by Rational Interpolation, (in) Proc. Colloquium on Complex Analysis, Joensuu, Finland, 1978, Springer Lecture Notes in Mathematics, Vol. 747, SpringerVerlag, Berlin, 1979, pp. 434-450.

[35] P. Wynn, A General System of Orthogonal Polynomials, Quart. J. Math. Oxford Ser., 18(1967), 81-96.

School of Mathematics

Georgia Institute of Technology

Atlanta, GA 30332-0160 USA

Email address: lubinsky@math.gatech.edu 